\title{
Correction
}

\section{Correction: Melo-Carrillo et al., "Selective Inhibition of Trigeminovascular Neurons by Fremanezumab: A Humanized Monoclonal Anti-CGRP Antibody"}

In the article "Selective Inhibition of Trigeminovascular Neurons by Fremanezumab: A Humanized Monoclonal Anti-CGRP Antibody” by Agustin Melo-Carrillo, Rodrigo Noseda, Rony Nir, Aaron J. Schain, Jennifer Stratton, Andrew M. Strassman, and Rami Burstein, which appeared on pages 7149-7163 of the July 26, 2017 issue, the third author's first name is incorrect. The corrected author list is as follows: Agustin Melo-Carrillo, Rodrigo Noseda, Rony-Reuven Nir, Aaron J. Schain, Jennifer Stratton, Andrew M. Strassman, and Rami Burstein. The author line has been corrected on the online PDF version.

DOI: 10.1523/JNEUROSCI.3012-17.2017 